\title{
Auditoria de enfermagem e a qualidade da assistência à saúde: uma revisão integrativa da literatura
}

\author{
Nursing audit and the quality of health care: an integrative literature review \\ Auditoría de enfermería y calidad de la atención sanitaria: revisión integradora de la literatura
}

\begin{abstract}
RESUMO
Nas últimas décadas, com o propósito de garantir a qualidade dos serviços prestados, as instituições de saúde têm-se preocupado em utilizar a auditoria, como ferramenta de forma contínua em suas organizações. Este estudo teve como objetivo analisar as produções científicas nacionais dos últimos 5 anos, sobre a qualidade da assistência de enfermagem através da auditoria, publicadas no banco de dado da Scielo (Scientific Electronic Library Online), Lilacs (Literatura Latino-Americana e do Caribe em Ciências da Saúde). Trata-se de um estudo de Revisão Integrativa da literatura. Em um primeiro momento da coleta, foram identificados 32 artigos dos quais foram selecionados apenas 8 artigos que responderam ao objetivo deste estudo. Através da análise dos artigos, foi possível identificar que a efetivação da auditoria de enfermagem facilita a avaliação da qualidade da assistência prestada ao cliente oferecendo condições para a sua melhoria e se torna uma ferramenta eficiente e imprescindível para o cuidado holístico.
\end{abstract}

DESCRITORES: Auditoria de enfermagem. Qualidade da assistência à saúde.

\section{ABSTRACT}

In recent decades, in order to guarantee the quality of the services provided, health institutions have been concerned with using auditing as a tool in their organizations on an ongoing basis. This study aimed to analyze the national scientific productions of the last 5 years, on the quality of nursing care through the audit, published in the database of Scielo (Scientific Electronic Library Online), Lilacs (Latin American and Caribbean Literature in Health Sciences). This is an integrative literature review study. In the first moment of the collection, 32 articles were identified, of which only 8 articles were selected that responded to the objective of this study. Through the analysis of the articles, it was possible to identify that the effectiveness of the nursing audit facilitates the assessment of the quality of the assistance provided to the client, offering conditions for its improvement and becomes an efficient and essential tool for holistic care.

DESCRIPTORS: Nursing audit. Quality of health care.

\section{RESUMEN}

En las últimas décadas, para garantizar la calidad de los servicios prestados, las instituciones de salud se han preocupado por utilizar la auditoría como herramienta en sus organizaciones de manera permanente. Este estudio tuvo como objetivo analizar las producciones científicas nacionales de los últimos 5 años, sobre la calidad de la atención de enfermería a través de la auditoría, publicadas en la base de datos de Scielo (Scientific Electronic Library Online), Lilacs (Literatura Latinoamericana y Caribeña en Ciencias de la Salud). Este es un estudio de revisión de literatura integradora. En el primer momento de la recopilación se identificaron 32 artículos, de los cuales se seleccionaron solo 8 artículos que respondieron al objetivo de este estudio. A través del análisis de los artículos, se pudo identificar que la efectividad de la auditoría de enfermería facilita la evaluación de la calidad de la asistencia brindada al cliente, ofreciendo condiciones para su mejora y se convierte en una herramienta eficiente y esencial para el cuidado integral. DESCRIPTORES: Auditoría de enfermería. Calidad de la asistencia sanitaria.

RECEBIDO EM: 30/07/2020 APROVADO EM: 27/08/2020

\section{Noely Machado Vieira}

Enfermeira Especialista em Gestão Estratégica na área da Saúde e em Auditoria em Saúde. Enfermeira da Vigilância Epidemiológica SMS- Cáceres. Preceptora da UNEMAT do estágio curricular II.

ORCID: 0000-0002-3705-7006 


\section{Bianca Teshima de Alencar}

Enfermeira Mestra em Ciências Ambientais-UNEMAT. Docente interina da UNEMAT na disciplina de Farmacologia. ORCID: 0000-0001-6812-3494

\section{Débora dos Santos Pinto}

Enfermeira Assistencial UBS- Santos Dumont - SMS- Cáceres. Especialista em Urgência e Emergência. Docente interina da UNEMAT na disciplina de Políticas de Saúde. Tutora do curso Bacharelado em Enfermagem UNOPAR.

ORCID: 0000-0002-1080-9167

\section{Dyone Rocha Teotonio}

Enfermeira efetiva da SMS-Cáceres, especialista em UTI-Neonatal e Gestão Hospitalar e Auditoria em Saúde. ORCID: 0000-0002-8514-2299

\section{Rosangela da Silva Conceição}

Enfermeira Especialista em Centro Cirúrgico e CME. Docente interina da UNEMAT. Coordenadora do trauma e emergência do Hospital Regional Dr. Antônio Fontes - Cáceres.

ORCID: 0000-0003-1622-4052

\section{INTRODUÇÃO}

D evido ao atual processo de globalização os setores de saúde tiveram que procurar novas alternativas para proporcionar a melhoria da gestão, como maneira das organizaçôes de saúde em adaptar-se a um mercado cada vez mais competitivo. Diante disso, houve a necessidade de garantir resultados positivos e clientes satisfeitos, exigindo assim que as organizações aprendam a associar baixos custos com excelência de qualidade para os seus clientes ${ }^{(1)}$.

Esta tendência mundial tem exigido dos profissionais envolvidos habilidade na análise de custos para a prestação de serviços de saúde visando desta forma um atendimento eficaz ${ }^{(2)}$.

Berti e Almeida (2005), relatam que os serviços de atenção à saúde buscam atender com a melhor qualidade possível seus clientes, ou seja, com o máximo de efetividade, eficiência, equidade, aceitabilidade, acessibilidade e adequabilidade ${ }^{(3)}$.

Atualmente as instituições de saúde, usufruem da auditoria como uma importante ferramenta para mensuração da qualidade e custos ${ }^{(12)}$. O processo de auditoria tem como conceito, a avaliação sistemática e formal de uma atividade realizada por pessoas não envolvidas diretamente em sua execução, a fim de determinar se a atividade está atendendo os objetivos propostos. Sendo possível assim desta forma, evidenciar falhas nas atividades desenvolvidas e apontar alternativas preventivas e corretivas para as mesmas $^{(4)}$.

A auditoria de enfermagem é uma área ainda pouco investigada, que serve como um instrumento de avaliação da qualidade da assistência nas instituições de serviços de saúde e propicia à melhoria do gerenciamento do cuidado de enfermagem, questiona-se então: Qual a produção científica nacional acerca de auditoria em enfermagem e sua repercussão perante a qualidade e desenvolvimento da assistência de enfermagem para o cuidado prestado aos usuários destes serviços?

Justifica-se a realização deste estudo, pois tem o intuito de relacionar os impactos da auditoria na qualidade da assistência, uma temática emergente no contexto dos serviços de saúde, pois através deste, pode-se observar e acompanhar a qualidade da assistência de enfermagem e se os gastos estão sendo condizentes com os procedimentos realizados dentro das instituições, a função administrativa do enfermeiro, sobretudo a função de liderança junto à equipe de enfermagem, propiciando desta forma uma atenção mais justa e humanizada do tratamento aos seus clientes e, além disso, o conhecimento produzido poderá fomentar futuras pesquisas e ações de melhorias que contribuíram para a atualização e melho- ria dos serviços da enfermagem.

Portanto, este estudo teve como objetivo analisar produções científicas nacionais dos últimos 5 anos, sobre a qualidade da assistência de enfermagem através da auditoria, publicadas no banco de dado da Scielo (Scientific Electronic Library Online), Lilacs (Literatura Latino-Americana e do Caribe em Ciências da Saúde) no período de 2014 a 2019, analisando a contribuição destes na melhoria da qualidade da assistência do enfermeiro nessa área, visto que atualmente ocorre uma escassez de produção cientifica dentro desta área.

\section{METÓDO}

O método elegido para o desenvolvimento do estudo em questão, foi a Revisão integrativa da literatura, pois o mesmo tem a finalidade de reunir e sintetizar resultados de pesquisas sobre um delimitado tema ou questão, ordenando e sistematizando o estudo, possibilitando o aprofundamento do conhecimento do assunto investigado ${ }^{(5)}$.

A pesquisa foi realizada durante o mês de julho a outubro de 2019. Foram utilizados os periódicos científicos nacionais disponíveis na integra encontrados nos sites da Scientific Electronic Library Online (Scielo), Literatura Latino-Americana e do Caribe em Ciências da Saúde (Lilacs), que apresentam periódicos na- 
cionais com artigos pertinentes ao tema. Para estabelecer a amostra que atendesse o objetivo do estudo foi utilizado o des-

critor: "Auditoria em enfermagem". Os critérios de inclusão foram os artigos estarem disponíveis na íntegra, entre os

\section{Quadro 1. Desenho do processo da busca pelos artigos:}

\section{Autoria em}

enfermagem

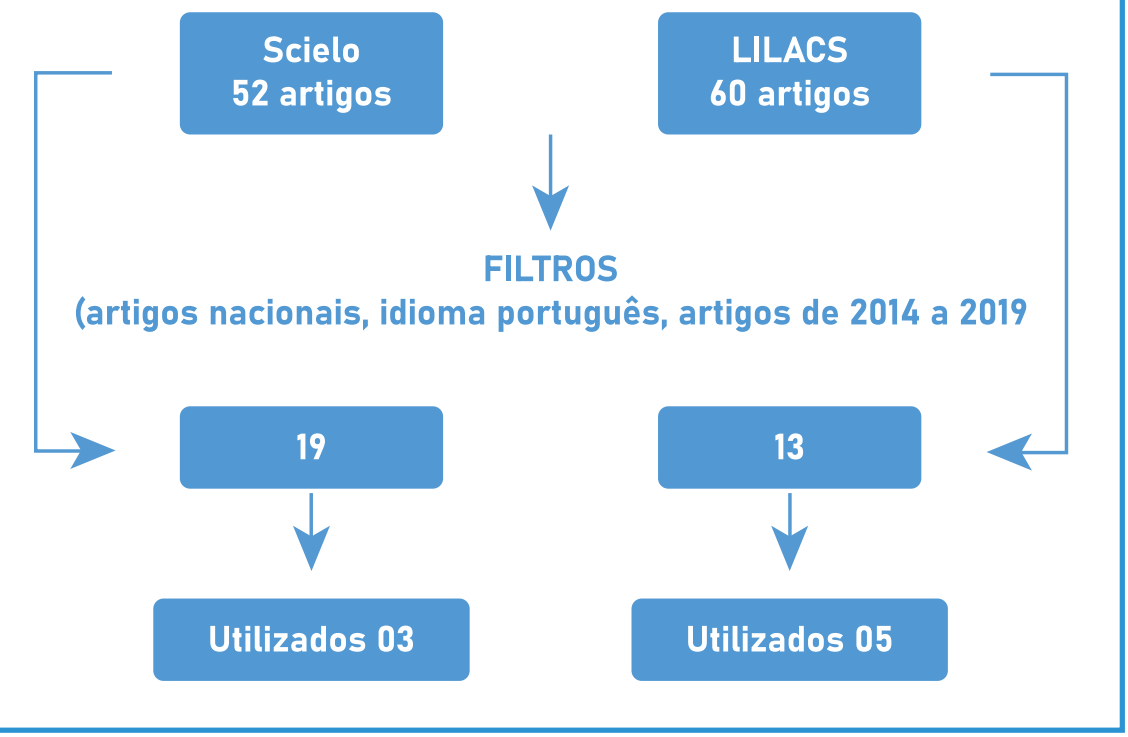

Fonte: dados da pesquisa. anos de 2014 a 2019, publicados nacionalmente e que trabalhassem dentro da temática elegida. Os critérios de exclusão, foram aqueles que no decorrer da leitura não dispunham de nenhuma contribuição relacionado a temática, estar fora do limite temporal estabelecido; idiomas diversos do requerido.

No quadro abaixo é possível observar como procedeu as buscas nos periódicos:

\section{RESULTADOS E DISCUSSAO}

Após a busca, foram selecionados 08 artigos, com relevância para o estudo em questão, para que seus resultados fossem analisados e interpretados, constituindo assim a amostra da pesquisa. Para tal, foi elaborado um instrumento de coleta de dados, contendo as seguintes variáveis: titulo do artigo, revista, ano de publicação, tipo de estudo, objetivo e conclusão. Conforme elaborado na tabela abaixo:

Após a análise dos artigos, foi possível agrupa-los em categorias temáticas, como acima já descritas, sendo elas: Qualidade da assistência de enfermagem, Registros de enfermagem e glosas.

\section{Tabela 2. Distribuição dos artigos selecionados para o estudo:}

\begin{tabular}{|c|c|c|c|c|c|c|}
\hline Temática & Título & Revista & $\begin{array}{c}\text { Ano de } \\
\text { publicação }\end{array}$ & Tipo de estudo & $\begin{array}{l}\text { Objetivo do } \\
\text { estudo }\end{array}$ & Conclusão \\
\hline Glosas & $\begin{array}{l}\text { Estudo da audi- } \\
\text { toria de contas } \\
\text { em um hospital } \\
\text { de ensino }\end{array}$ & $\begin{array}{l}\text { Revista Brasilei- } \\
\text { ra de Enferma- } \\
\text { gem }\end{array}$ & 2015 & $\begin{array}{l}\text { pesquisa quan- } \\
\text { titativa explora- } \\
\text { tória, descritiva, } \\
\text { do tipo estudo de } \\
\text { caso único. }\end{array}$ & $\begin{array}{l}\text { verificar os itens } \\
\text { componentes das } \\
\text { contas hospitala- } \\
\text { res, conferidos por } \\
\text { enfermeiros audi- } \\
\text { tores }\end{array}$ & $\begin{array}{l}\text { Considera-se que este } \\
\text { estudo possa represen- } \\
\text { tar a possibilidade de } \\
\text { avanço no conhecimen- } \\
\text { to da auditoria de contas } \\
\text { hospitalares }\end{array}$ \\
\hline $\begin{array}{l}\text { Qualidade da } \\
\text { assistência de } \\
\text { enfermagem }\end{array}$ & $\begin{array}{l}\text { Implantação da } \\
\text { auditoria concor- } \\
\text { rente de enfer- } \\
\text { magem: um rela- } \\
\text { to de experiência }\end{array}$ & $\begin{array}{l}\text { Texto \& Contex- } \\
\text { to- Enferma- } \\
\text { gem }\end{array}$ & 2016 & $\begin{array}{l}\text { Relato de experi- } \\
\text { ência }\end{array}$ & $\begin{array}{l}\text { descrever a expe- } \\
\text { riência de enfer- } \\
\text { meiras acerca da } \\
\text { implantação da } \\
\text { auditoria concor- } \\
\text { rente de enferma- } \\
\text { gem em ambiente } \\
\text { hospitalar. }\end{array}$ & $\begin{array}{l}\text { Os resultados apontam a } \\
\text { viabilidade de se adotar } \\
\text { este modelo e benefí- } \\
\text { cios, principalmente no } \\
\text { que tange a diminuição } \\
\text { do tempo para envio da } \\
\text { fatura aos convênios mé- } \\
\text { dicos, maior interação en- } \\
\text { tre as auditoras e equipes } \\
\text { assistenciais, criação de } \\
\text { indicadores, e contribui- } \\
\text { ções para a melhoria da } \\
\text { qualidade assistencial e } \\
\text { registros de enfermagem. }\end{array}$ \\
\hline
\end{tabular}




\section{artigo}

\begin{tabular}{|c|c|c|c|c|c|c|}
\hline $\begin{array}{l}\text { Qualidade da } \\
\text { assistência de } \\
\text { enfermagem }\end{array}$ & $\begin{array}{l}\text { Auditoria de prá- } \\
\text { ticas de precau- } \\
\text { ções-padrão e } \\
\text { contato em Uni- } \\
\text { dade de Terapia } \\
\text { Intensiva }\end{array}$ & $\begin{array}{l}\text { Revista da esco- } \\
\text { la de enferma- } \\
\text { gem da USP }\end{array}$ & 2019 & $\begin{array}{l}\text { Estudo descriti- } \\
\text { vo, transversal e } \\
\text { prospectivo. }\end{array}$ & $\begin{array}{l}\text { Avaliar a estrutu- } \\
\text { ra e a adesão às } \\
\text { medidas de pre- } \\
\text { cauções-padrão } \\
\text { e específicas dos } \\
\text { profissionais de } \\
\text { saúde em Unidade } \\
\text { de Terapia Intensi- } \\
\text { va de hospital de } \\
\text { ensino, no DF. }\end{array}$ & $\begin{array}{l}\text { Verificou-se boa adesão } \\
\text { ao uso de luvas, avental e } \\
\text { máscara, baixa adesão ao } \\
\text { uso de óculos de proteção } \\
\text { e uso desnecessário de } \\
\text { máscaras e precauções } \\
\text { de contato admissionais. }\end{array}$ \\
\hline $\begin{array}{l}\text { Registros de } \\
\text { enfermagem }\end{array}$ & $\begin{array}{l}\text { A elaboração de } \\
\text { evoluções de } \\
\text { enfermagem e } \\
\text { possíveis dificul- } \\
\text { dades: percep- } \\
\text { ção do enfer- } \\
\text { meiro }\end{array}$ & Revista Nursing & 2019 & $\begin{array}{l}\text { Descritivo e quali- } \\
\text { tativo }\end{array}$ & $\begin{array}{l}\text { Analisar a percep- } \\
\text { ção dos enfermei- } \\
\text { ros de um hospital } \\
\text { público de Belém, } \\
\text { acerca das possí- } \\
\text { veis dificuldades } \\
\text { para a elaborações } \\
\text { de evoluções es- } \\
\text { critas. }\end{array}$ & $\begin{array}{l}\text { A falta de tempo, é o } \\
\text { principal dificultador } \\
\text { para a realização da evo- } \\
\text { lução de enfermagem } \\
\text { em todos os aspectos } \\
\text { que deve contemplar. }\end{array}$ \\
\hline $\begin{array}{l}\text { Registros de } \\
\text { enfermagem }\end{array}$ & $\begin{array}{l}\text { Auditoria da } \\
\text { qualidade dos } \\
\text { registros de en- } \\
\text { fermagem em } \\
\text { prontuários em } \\
\text { um hospital uni- } \\
\text { versitário }\end{array}$ & $\begin{array}{l}\text { Enfermagem } \\
\text { Foco }\end{array}$ & 2019 & $\begin{array}{l}\text { Pesquisa descriti- } \\
\text { va, quantitativa }\end{array}$ & $\begin{array}{l}\text { Analisar a qualida- } \\
\text { de dos registros de } \\
\text { enfermagem em } \\
\text { prontuários. }\end{array}$ & $\begin{array}{l}\text { Os resultados sugerem } \\
\text { necessidade de cons- } \\
\text { tante atuação dos pro- } \\
\text { gramas educacionais } \\
\text { para o fortalecimento de } \\
\text { ações que visem a exce- } \\
\text { lência dos registros de } \\
\text { enfermagem. }\end{array}$ \\
\hline $\begin{array}{l}\text { Registros de } \\
\text { enfermagem }\end{array}$ & $\begin{array}{l}\text { Registros de en- } \\
\text { fermagem: uma } \\
\text { revisão integra- } \\
\text { tiva da literatura }\end{array}$ & Revista nursing & 2018 & $\begin{array}{l}\text { Revisão integrati- } \\
\text { va da literatura }\end{array}$ & $\begin{array}{l}\text { Descrever a pro- } \\
\text { dução cientifica, } \\
\text { sobre as princi- } \\
\text { pais informações } \\
\text { encontradas nos } \\
\text { registros de enfer- } \\
\text { magem de } 2005 \text { a } \\
2015 \text {. }\end{array}$ & $\begin{array}{l}\text { Concluiu-se a necessida- } \\
\text { de de investimentos em } \\
\text { educação permanente e } \\
\text { chamar a atenção para o } \\
\text { que deve ser considerado } \\
\text { essencial nas evoluções. }\end{array}$ \\
\hline $\begin{array}{l}\text { Qualidade da } \\
\text { assistência de } \\
\text { enfermagem }\end{array}$ & $\begin{array}{l}\text { Auditoria de en- } \\
\text { fermagem: revi- } \\
\text { são integrativa } \\
\text { da literatura }\end{array}$ & Revista nursing & 2018 & $\begin{array}{l}\text { Revisão integrati- } \\
\text { va da literatura }\end{array}$ & $\begin{array}{l}\text { Identificar as evi- } \\
\text { dencias cientificas } \\
\text { atuais da auditoria } \\
\text { no campo da en- } \\
\text { fermagem e discu- } \\
\text { ti-las. }\end{array}$ & $\begin{array}{l}\text { Concluiu-se que a ne- } \\
\text { cessidade de mais pes- } \\
\text { quisas que abordem a } \\
\text { experiência das enfer- } \\
\text { meiras auditoras, sua } \\
\text { rotina, dificuldades e } \\
\text { necessidades, para um } \\
\text { saber pautado na cienti- } \\
\text { ficidade e na pratica. }\end{array}$ \\
\hline $\begin{array}{l}\text { Registros de } \\
\text { enfermagem }\end{array}$ & $\begin{array}{l}\text { Inconsistências } \\
\text { das anotações } \\
\text { de enfermagem } \\
\text { no processo de } \\
\text { Auditoria }\end{array}$ & $\begin{array}{l}\text { Revista de en- } \\
\text { fermagem do } \\
\text { centro oeste mi- } \\
\text { neiro }\end{array}$ & 2016 & $\begin{array}{l}\text { descritivo, reali- } \\
\text { zado por meio de } \\
\text { uma revisão inte- } \\
\text { grativa da litera- } \\
\text { tura. }\end{array}$ & $\begin{array}{l}\text { identificar as prin- } \\
\text { cipais falhas nas } \\
\text { anotações de en- } \\
\text { fermagem dos } \\
\text { prontuários dos } \\
\text { pacientes. }\end{array}$ & $\begin{array}{l}\text { é necessário investir em } \\
\text { capacitação dos profis- } \\
\text { sionais da saúde buscan- } \\
\text { do qualidade nas ano- } \\
\text { tações de enfermagem } \\
\text { para que se possa dar } \\
\text { continuidade ao cuidado, } \\
\text { bem como obter respaldo } \\
\text { ético e legal diante dos } \\
\text { aspectos jurídicos. }\end{array}$ \\
\hline
\end{tabular}


Qualidade da assistência de enfermagem

A auditora dentro da área da saúde, tem por objetivo obter informações sobre o controle da qualidade dos serviços e possibilita subsídios para buscar maior eficiência dentro do gerenciamento das ações de saúde ${ }^{(6)}$.

É através da auditoria, possível verificar a qualidade da assistência prestada aos pacientes, o que contribui para a sua constante melhoria e aperfeiçoamento de técnicas. Ultimamente ela vem ganhando notoriedade dentro da gestão hospitalar devido unir a melhoria do cuidado e analise de contas hospitalares ${ }^{(7,12)}$.

A expansão no mercado e trabalho na função do enfermeiro auditor é promissora, principalmente dentro das análises das contas hospitalares, pois ele realiza a compatibilidade dos custos com as ações realizadas e o material consumido ${ }^{(7)}$.

Para Barreto et al. (2016), a assistência e os cuidados prestados embasados em recursos preestabelecidos pode ser mudada sempre que necessário, após a avaliação criteriosa da qualidade da auditoria em enfermagem ${ }^{(8)}$.

Um dos grandes benefícios da auditoria para a instituição, é poder verificar se houve o correto planejamento das ações e planos pretendidos, podendo ser reformulado e direcionado visando melhorias dos controles dos custos ${ }^{(8)}$.

Após o processo de análise dos artigos, pode-se observar que a auditoria em enfermagem é um processo exigente, requer dinamização constante de toda equipe de enfermagem, atenção sempre voltada para o desempenho das ações de enfermagem, integração na equipe, visando interesses direcionados num único objetivo a qualidade da assistência.

\section{Registros de enfermagem}

Dentre as profissóes do hospital, a enfermagem é a que permanece por um longo período ao lado do paciente, atendendo-o de uma forma humanizada e holística, o tornando assim o profissional responsável pela maioria das informações referentes ao cuidado do paciente. Desta

\section{Diante disso,}

os registros no

prontuário servem

como elementos

primordiais para

estas avaliaçôes,

e igualmente

subsidiam a

comunicação entre

os profissionais

de saúde. São

documentos de

grande importância

para auditoria

da qualidade e

asseguram o serviço

prestado aos clientes. forma se torna indispensável o registro destas informações ${ }^{(9)}$.

O art. $6^{\circ}$ da Resolução do Conselho Federal de Enfermagem (COFEN), diz que "a execução do processo de enfermagem deve ser registrada formalmente". Os registros de enfermagem constituem uma forma de comunicação escrita entre os membros responsáveis pelo paciente, de maneira a proporcionar a continuidade do cuidado ${ }^{(9,10)}$.

De acordo com Silva et al. (2019), o registro de enfermagem constitui ainda parte integrante e indispensável da Sistematização da Assistência de Enfermagem (SAE), tornando assim um processo continuo de assistência individualizada e como documento legal de amparo judicial, caso seja necessário futuramente ${ }^{(9)}$.

Os registros realizados de forma adequada, visam não só a continuidade da assistência como um todo, mas também fornecem embasamento cientifico para pesquisas e o processo de auditoria ${ }^{(9)}$.

Para que o processo seja desenvolvido da forma correta é necessário que seja seguido embasado em regras, dentre elas: ser claro e coerente, referir data e horário do procedimento desenvolvido, conter informações fornecidas e obtidas ${ }^{(9)}$.

Um dos grandes problemas encontrados dentro da auditoria é a inconformidade de registros no prontuário dos pacientes, o que pode ocasionar discrepâncias nas cobranças hospitalares e indicar falhas na assistência, é de fundamental importância que o enfermeiro auditor realize diálogos orientativos com a equipe para que o problema seja sanado ${ }^{(7)}$.

Diante disso, os registros no prontuário servem como elementos primordiais para estas avaliações, e igualmente subsidiam a comunicação entre os profissionais de saúde. São documentos de grande importância para a auditoria da qualidade e asseguram o serviço prestado aos clientes.

\section{Glosas}

Em seu estudo Lima et al. (2018), define glosa como o não pagamento total ou parcial, dos itens de uma fatura, ou 


\section{artigo}

Vieira, N.M.; Alencar, D.T.; Pinto, D.S.; Teotonio, D.R.; Conceição, R.S.

Auditoria de enfermagem e a qualidade da assistência à saúde: uma revisão integrativa da literatura

dela por completo. Evidencia ainda, que a glosa pode ocorrer por diversos motivos, dentre eles a não autorização de medicamentos ou procedimentos, ou pela não utilização do material cobrado ${ }^{(11)}$.

É classificada em dois tipos, conforme a situação encontrada, pode ser técnica relacionada a procedimentos médicos e da enfermagem; e administrativa - relacionada a inconformidades ${ }^{(11)}$.

As falhas ocasionadas pelos registros mal realizados, provocam conflitos e divergem informações sobre os procedimentos executados, originando desta forma as glosas hospitalares, causando prejuízo para os cuidados prestados pelos profissionais enfermeiros e para o fornecimento de informações que possam amparar o profissional ou a instituição judicialmente ${ }^{(9,12)}$.

Os Resultados das pesquisas apontam que os registros de enfermagem contribuem substancialmente para um alto percentual de recuperação dos itens glosados. Os autores evidenciam que existem as grandes falhas nos registros de enfer- magem e os mesmos ocasionam prejuízos as instituições de saúde.

\section{CONCLUSÃO}

Fica evidente a importância do trabalho do profissional enfermeiro auditor, pois além ser uma tendência de mercado é um ramo em ascensão, em suas diversas vertentes. Possibilitando o enfermeiro agir como auditor de contas, da qualidade da assistência, em pesquisas e processos. A valorização do enfermeiro auditor é uma realidade nas instituições hospitalares que visam neste profissional à consolidação do atendimento prestado por suas equipes.

É visível o prejuízo financeiro quando há inconformidades nos procedimentos realizados ou ausência de anotações nos prontuários pela equipe de enfermagem, uma vez que pode ser observado pelo alto número de glosas apresentadas pelas operadoras junto às instituições de saúde.

A auditoria pode ser considerada um elemento essencial para mensurar a qua- lidade da assistência de Enfermagem, oferecendo subsídios aos profissionais para orientar suas atividades, estimulando a reflexão individual e coletiva e nortear o processo de educação permanente, concomitante a este processo possibilita a redução das glosas.

Desta forma foi possível concluir que os resultados dos estudos descrevem as principais distorções e não conformidades presentes nas anotações de enfermagem, relacionando-as, principalmente, com a qualidade da assistência de enfermagem e os prejuízos financeiros à instituição. Também destacam-se a função administrativa do enfermeiro, sobretudo a sua função de liderança junto à equipe de enfermagem e a auditoria de enfermagem como um processo educativo e de fundamental importância a inclusão dessa temática dentro dos currículos institucionais, preparando os profissionais para desempenhar a função de auditor nos serviços de enfermagem coordenando e gerindo as equipes de saúde.

\section{REFERÊNCIAS}

1. Silva KR, Lima MDO, Sousa MA. Auditoria: ferramenta de enfermagem para melhoria da qualidade assistencial. Rev. Eletrônica Gestão \& Sáude 2016.

2. Paim CRP, Ciconelli RM. Auditoria de avaliação da qualidade dos serviços de saúde. Revista de Administração em Saúde. 2007;9(36):85-91.

3. Berti HW, Almeida ES. Percepção dos gestores municipais de saúde sobre qualidade da assistência e controle social. Revista Nursing. 2005;88(8):425-3.

4. Siqueira PLF. Auditoria em saude e atribuições do enfermeiro auditor. Cad. Saúde Desenvolv [internet].2014 [citado 2018 mar.29]; 3 (2):1-19.

5. Mendes KDS, Silveira RCCP, Galvão CM. Revisão integrativa: método de pesquisa para a incorporação de evidencias na saúde e na enfermagem. Texto completo Enferm. 2008 Out-Dez;17(4):758-64.

6. Castro AF, Rodrigues MCS. Auditoria de práticas de precauções-padrão e contato em Unidade de Terapia Intensiva. Rev. esc. enferm. USP vol.53 São Paulo 2019 Epub Aug 19,2019.

7. Viana CD, Bragas LZT, Lazarri DD, Garcia CTF, Moura GMSS. Implantação da auditoria concorrente de Enfermagem: um relato de experiência. Texto contexto - enferm. vol.25 no.1 Florianópo- lis 2016 Epub Apr 01, 2016.

8. Barreto JA, Lima GG, Xavier CF. Inconsistências das anotações de Enfermagem no processo de Auditoria. R. Enferm. Cent. O. Min. 2016 jan/abr; 1(6):2081-2093.

9. Silva, AGI, Dias BRL, Leite MR. A elaboração de evoluções de enfermagem e possíveis dificuldades: percepção do enfermeiro. Revista Nursing, 2019; 22 (254):3039-3042.

10. Conselho Federal de Enfermagem (COFEN). Guia de Recomendações para Registro de Enfermagem no Prontuário do Paciente e outros Documentos de Enfermagem. Portaria n. 523/2015 [Internet]. 2016 [cited 2018 Out 29]. Available from: http://www. cofen.gov.br/wp-content/uploads/2016/08/Guia-de-Recomenda\%C3\%A7\%C3\%B5es-CTLN-Vers\%C3\%A3o-Web. pdf.

11. Lima RJ, Pimenta CJL, Frazão MCLO, Silva CRR, Viana LRC, Salviano GR. Auditoria de enfermagem: revisão integrativa da literatura. Revista Nusing, 2018; 21 (247): 2531-2534.

12. Silva VA, Mota RS, Oliveira LS, Jesus N, Carvalho CM, Magalhães LGS. Auditoria da qualidade dos registros de enfermagem em prontuários em um hospital universitário. Enferm. Foco 2019; 10 (3): 28-33. 